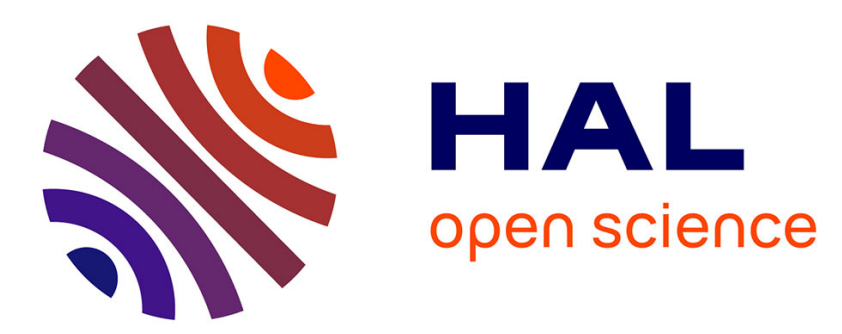

\title{
Characterization and photooxidative behaviour of nanocomposites formed with polystyrene and LDHs organo-modified by monomer surfactant.
}

Jean-Luc Gardette, Bénédicte Mailhot, Sandrine Morlat-Therias, Fabrice Leroux, Lynda Meddar

\section{To cite this version:}

Jean-Luc Gardette, Bénédicte Mailhot, Sandrine Morlat-Therias, Fabrice Leroux, Lynda Meddar. Characterization and photooxidative behaviour of nanocomposites formed with polystyrene and LDHs organo-modified by monomer surfactant.. Polymer, 2005, 46, pp.3571-3578. hal-00015628

\section{HAL Id: hal-00015628 \\ https://hal.science/hal-00015628}

Submitted on 14 Feb 2007

HAL is a multi-disciplinary open access archive for the deposit and dissemination of scientific research documents, whether they are published or not. The documents may come from teaching and research institutions in France or abroad, or from public or private research centers.
L'archive ouverte pluridisciplinaire $\mathbf{H A L}$, est destinée au dépôt et à la diffusion de documents scientifiques de niveau recherche, publiés ou non, émanant des établissements d'enseignement et de recherche français ou étrangers, des laboratoires publics ou privés. 


\title{
Characterization and photooxidative behaviour of nanocomposites formed with polystyrene and LDHs organo-modified by monomer surfactant
}

\author{
Fabrice Leroux $^{\mathrm{a}, *}$, Lynda Meddar ${ }^{\mathrm{a}}$, Bénédicte Mailhot ${ }^{\mathrm{b}}$, Sandrine Morlat-Thérias ${ }^{\mathrm{b}}$, \\ Jean-Luc Gardette ${ }^{\mathrm{b}}$ \\ ${ }^{\mathrm{a}}$ Laboratoire des Matériaux Inorganiques, UMR CNRS-UBP 6002, Université Blaise Pascal (Clermont-Ferrand), 24 av. des Landais, \\ 63177 Aubière, Cedex, France \\ ${ }^{\mathrm{b}}$ Laboratoire de Photochimie Moléculaire et Macromoléculaire, UMR CNRS-UBP 6505, Université Blaise Pascal (Clermont-Ferrand), \\ 24 av. des Landais, 63177 Aubière, Cedex, France
}

Received 14 February 2005; received in revised form 16 March 2005; accepted 17 March 2005

\begin{abstract}
Layered double hydroxide (LDH) organo-modified by a surface-active monomer, 3-sulfopropyl methacrylate (SPMA) was used as filler for polystyrene (PS). Different nanocomposites SPMA/LDH:PS were prepared by bulk polymerization process using low amount of initiator. The two components, filler and polymer, as well as the degree dispersion of the reactive filler as a function of its loading in PS were characterized by a combination of several techniques: X-ray diffraction, high resolution ${ }^{13} \mathrm{C}$ CP-MAS NMR, FTIR, UV-visible, thermal analysis, SEC and MET. The nanocomposites were submitted to UV-light exposures in the presence of oxygen. The oxidation photoproducts and the rates of oxidation were compared for the various samples. It was shown that the filler was not modifying the oxidation mechanism of the polymer, but had a slight effect on the oxidation rate.
\end{abstract}

(C) 2005 Elsevier Ltd. All rights reserved.

Keywords: LDH reactive nanofiller; Polystyrene; Nanocomposites

\section{Introduction}

Inherent to the enhanced properties observed in terms of mechanical reinforcement, gas barriers and fire-retardancy, there is an ever-growing interest in polymer nanocomposites [1-3]. However, additional to the achievement of such properties, the durability under specific conditions of use (outdoor weathering) should be strongly considered as it is an important prerequisite for a nanocomposite to find potential applications. It is well known that organic polymers may degrade when exposed to UV-light in the presence of air, and a question arises whether the addition of filler is accelerating oxidative processes. Conversely, any impediment in the time alteration would be of great interest. Indeed, among the literature related to nanocomposite

\footnotetext{
* Corresponding author. Tel.: +1 133473 407036; fax: +1 133473 407108.

E-mail address: fleroux@chimtp.univ-bpclermont.fr (F. Leroux).
}

0032-3861/\$ - see front matter (C) 2005 Elsevier Ltd. All rights reserved. doi:10.1016/j.polymer.2005.03.044 materials, only few papers deal with photodegradation of polymer/clay nanocomposites [4-7], but exclusively related to montmorillonite as nanoclay in the so called polymerlayered silicate nanocomposites (PLS). Our previous results indicated that the photodegradation mechanism of the polymeric matrix was not modified by the fillers, but a dramatic influence of the clay on the rate of degradation was reported [8,9]. A decrease in photostability was observed, resulting from interactions with antioxidants.

Mostly focused on cationic clays, and particularly on montmorillonite and hectorite, smectite-type layered silicates, clay-based nanofillers have recently been extended to the family of layered double hydroxides (LDH).

Hydrotalcite-like LDH materials are described according to the ideal formula, $\left[\mathrm{M}_{1-x}^{\mathrm{II}} \mathrm{M}_{x}^{\mathrm{III}}(\mathrm{OH})_{2}\right]_{\text {intra }}^{x+}\left[\mathrm{A}_{x / m}^{m-} \cdot n \mathrm{H}_{2} \mathrm{O}\right]_{\text {inter }}$, where $\mathrm{M}^{\mathrm{II}}$ and $\mathrm{M}^{\mathrm{III}}$ are metallic cations, $A$ the anions, intra and inter denote the intralayer and interlayer domain, respectively. The structure consists of brucite-like layers constituted of edge-sharing octahedra. The presence of trivalent cations induces positive charges in the layers, which are counterbalanced by interlamellar anions. LDH materials are considered as promising host structure $[10,11]$. 
The dispersion of organo-modified LDH into various polymers such as epoxy [12] and poly(imide) [13] has been reported by Hsueh and Chen. The mechanical properties were fullfilling the expectations, with increase in tensile modulus of the nanocomposite with the nanofiller content.

In order to render the inorganic platelets organophilic, long chain organic molecules are usually used. This presents the advantage of proping apart the lamellae, thus helping for the exfoliation process. Although, it appears quite questionable if a complete exfoliation is wished [14] and, in some cases, ordered mixed intercalated and exfoliated structure may be preferred. Alternatively to the use of surfactant molecule as compatibilizer agent, an effective other approach consists in utilizing reactive organophilic fillers in order to achieve strong interactions between the two components of the nanocomposite. In this idea, the use of monomer surfactant may be of interest, having in mind that immobilisation of polymer chain should affect the glass transition temperature $T_{\mathrm{g}}$ as exemplified by the system polystyrene: montmorillonite organo-modified by a quaternary salt of the aminomethylstyrene [15].

In this study, we were interested in better understanding the interaction and subsequent oxidative behavior under UV-light exposure of nanocomposite using poly(styrene) (PS) as polymer and LDH organo-modified by a monomer surfactant as filler. Our choice was orientated by the fact that PS is widely used, and that its oxidative behavior is very well known [16,17], and that, to our knowledge, it is the first time that such hybrid lamellar system is employed as filler. The nature of dispersion and the photo-oxidation mechanisms of the formed nanocomposites were investigated by a combination of several techniques.

\section{Experimental section}

\subsection{Materials}

3-Sulfopropyl methacrylate potassium salt (SPMA, $\mathrm{H}_{2-}$ $\mathrm{C}=\mathrm{C}\left(\mathrm{CH}_{3}\right) \mathrm{CO}_{2}\left(\mathrm{CH}_{2}\right)_{3} \mathrm{SO}_{3} \mathrm{~K}$ ) (Aldrich, 98\%), $\mathrm{ZnCl}_{2}$, $\mathrm{Al}\left(\mathrm{NO}_{3}\right)_{3} \cdot 9 \mathrm{H}_{2} \mathrm{O}$ (Acros, 99\%), $\mathrm{NaOH}$ (Acros, $97 \%$ ), and benzoyl peroxide (BPO) (Aldrich, 97\%) were used as received. Styrene in presence of an inhibitor (1,2-dihydroxy 4-tertiobutylbenzene) and poly(styrene) standard $\left(M_{\mathrm{w}}=\right.$ $200,000 \mathrm{~g} / \mathrm{mol}$ ) were purchased from Aldrich. The monomer was purified by distillation under reduced pressure at $50{ }^{\circ} \mathrm{C}$.

\subsection{Preparation of the $L D H$ hybrid materials}

The cation composition of the $\mathrm{LDH}$ material is $\mathrm{Zn}_{2} \mathrm{Al}$, and the preparation of the hydrotalcite-like hybrid materials SPMA/LDH has been reported in a previous paper [17]. Briefly, the so-called coprecipitation method was used; experimentally, a solution of SPMA $\left(3 \times 10^{-2} \mathrm{M}\right)$ was prepared, and $250 \mathrm{ml}$ solution of the salts $\left(\mathrm{Zn}-2 \times 10^{-2}\right.$ and $\mathrm{Al}-1 \times 10^{-2} \mathrm{M}$ ) were added dropwise to the previous solution. During the addition, the reaction is kept under nitrogen atmosphere in order to avoid contamination by carbonate and the $\mathrm{pH}$ is kept constant with the addition of $\mathrm{NaOH}$. Contrary to our previous work, the coprecipitation time was performed in $10^{\prime}$ and the slurry is directly separated from the mother liquid by centrifugation rather than aged. The resulting powders hereafter named as SPMA/LDH were washed several times with distilled water and finally dried at room temperature.

\subsection{Preparation of the nanocomposites}

The nanocomposites were prepared by bulk polymerization using BPO as initiator. SPMA/LDH and BPO $(4.5 \mathrm{mg}-0.1 \% \mathrm{wt}$ ) was incorporated into $5 \mathrm{ml}$ of a distilled solution of styrene. The quantity of initiator decreases the size of the formed polymer, the use of $1 \%$ wt of BPO was giving rise to brittle polymer. Different loadings of filler (SPMA/LDH) were added to PS. Experimentally, as much as $679.5 \mathrm{mg}$ of SPMA/LDH (15\% wt loading) were dispersed into the styrene solution. The mixture was vigorously stirred for $4 \mathrm{~h}$ at room temperature, and then placed for $1 \mathrm{~h}$ under nitrogen atmosphere before the polymerization.

At this step, cloudy suspensions were found to be stable in styrene for a long time. The polymerization process was induced by heat at $80^{\circ} \mathrm{C}$ for $24 \mathrm{~h}$ for styrene (free of filler), whereas a temperature of $95{ }^{\circ} \mathrm{C}$ was needed for the nanocomposites to complete the polymerization. Remaining styrene was removed from the nanocomposite by a thermal treatment at $60{ }^{\circ} \mathrm{C}$ under vacuum.

Thin films were necessitated for photooxidation studies, in order to avoid oxygen starvation effects during exposure to UV-light in the presence of air, and also in order to facilitate quantitative analysis by infrared spectroscopy in transmission mode. The nanocomposite films were obtained by compression moulding between two hot plates $\left(180{ }^{\circ} \mathrm{C}\right.$ and 200 bars) for $1^{\prime}$. Films of 100-200 $\mu$ m thickness were obtained.

\subsection{Techniques of characterization}

Powder X-ray diffraction profiles (PXRD) were obtained with a Philipps X-Pert Pro X-ray diffractometer with a diffracted beam monochromator $\mathrm{Cu} \mathrm{K}_{\alpha}$ source, the counting time was of $4 \mathrm{~s}$ and the angle step of $0.04^{\circ}$.

Thermal gravimetric (TGA) and differential thermal analyses DTA were performed simultaneously and recorded with a Setaram TG-DTA92 thermogravimetric analyser at a typical rate of $5{ }^{\circ} \mathrm{C} / \mathrm{min}$ under air atmosphere.

The differential scanning calorimeter used to measure the glass transition $T_{\mathrm{g}}$ was a Mettler Toledo DSC30. The heating rate was $10^{\circ} \mathrm{C} / \mathrm{min}$ and the temperature range was from 25 to $180^{\circ} \mathrm{C}$.

UV-light irradiations at $\lambda>300 \mathrm{~nm}$ in presence of 
oxygen were performed using a SEPAP 12/24 unit at a temperature of $60{ }^{\circ} \mathrm{C}$. This apparatus has been designed for the study of polymer photodegradation in artificial conditions corresponding to a medium acceleration of ageing [18]. SEPAP 12/24 units are equipped with four medium pressure mercury lamps. A borosilicate envelope filters wavelengths below $300 \mathrm{~nm}$.

The hybrid LDH-SPMA was irradiated and characterized by infra-red in $\mathrm{KBr}$ pellets. Nanocomposite samples of PS/LDH were studied as films.

Infrared spectra were recorded in transmission mode with a Nicolet 760 Magna-FTIR spectrometer, working with OMNIC software. Spectra were obtained using 32 scans and a $4 \mathrm{~cm}^{-1}$ resolution.

In order to compare the spectra of different formulations of nanocomposites, a calibration of the thickness $(\mu \mathrm{m})$ of each sample was performed at $1583 \mathrm{~cm}^{-1}$ (aromatic stretch).

UV-visible spectra were recorded on a Shimadzu UV$2101 \mathrm{PC}$ spectrometer equipped with an integrating sphere.

${ }^{13} \mathrm{C}(I=1 / 2)$ solid state NMR experiments were performed with a 300 Bruker spectrometer at $75.47 \mathrm{MHz}$, using magic angle spinning (MAS) condition at $10 \mathrm{kHz}$ and a $4 \mathrm{~mm}$ diameter size zirconia rotor. ${ }^{13} \mathrm{C}$ spectra obtained by proton enhanced cross-polarization method (CP) are referenced to the carbonyl of the glycine calibrated at $176.03 \mathrm{ppm}$.

Size exclusion chromatography (SEC) measurements were carried out with a Viscotek Trisec 270 Controller as viscosimetric detector and a VE3580 Viscotek refractive index detector. The mobile phase was THF (Aldrich HPLC grad) with a flow rate of $1 \mathrm{ml} / \mathrm{min}$ and separation was achieved using a GMHxl column. The calibration curve was obtained using polystyrene standards. Average molecular weights $\left(M_{\mathrm{w}}\right.$ and $\left.M_{\mathrm{n}}\right)$ were calculated, as well as the polydispersity index $\left(I_{\mathrm{p}}=M_{\mathrm{w}} / M_{\mathrm{n}}\right)$. Experimentally, fillerfree polymers were solubilized in THF, and then directly injected, whereas prior to injection, the polymers obtained from the as made nanocomposites were obtained after a first dissolution in THF, then filtration and recrystallization in ethanol.

TEM images were obtained by using a Philips CM120 operating at an acceleration voltage of $80 \mathrm{kV}$. The samples were cut in thin lamella of $60-80 \mathrm{~nm}$ width by a microtome at $-80{ }^{\circ} \mathrm{C}$.

\section{Results and discussion}

\subsection{Crystallinity of the hybrid materials SPMA/LDH}

Having in mind that a large number of platelets may be of inconvenience for a lamellar structure to delaminate, we studied the crystallinity of the hybrid phases obtained after a rapid coprecipitation and by changing the value of $\mathrm{pH}$ from 11 down to 5. The XRD diagrams are displayed in Fig. 1. It

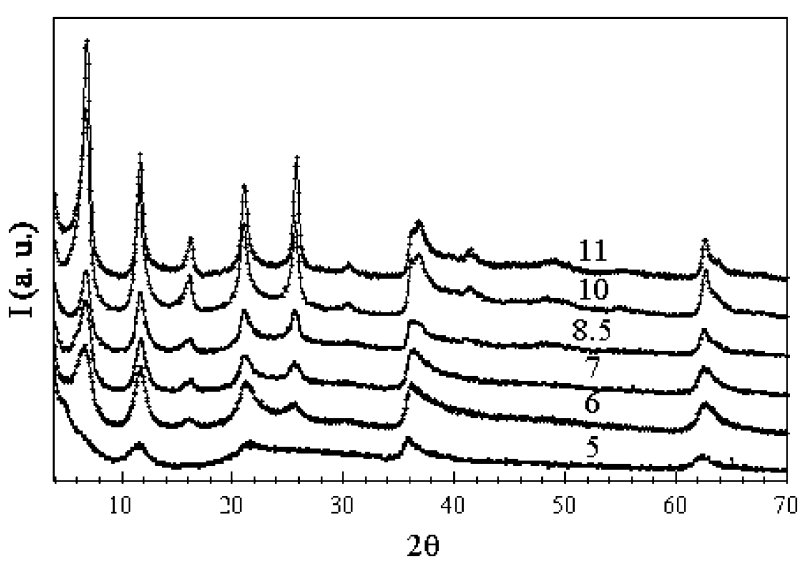

Fig. 1. XRD of the hybrid filler SPMA/LDH prepared by coprecipitation, the $\mathrm{pH}$ is indicated.

can be observed that a decreased in $\mathrm{pH}$ gives rise to less crystallised phases as the diffraction lines $(00 l)$ are strongly enlarged, but the positions are unchanged. It is interpreted by the formation of SPMA/LDH as previously evidenced [19], and it suggests that for all the phases, the accomodation of the organic molecule in the gap is similar.

A large broadening of the diffraction line (110) is also depicted, showing that the progressive loss of the stacking sequence is also associated to an in-plane structural disorder.

For the $\mathrm{pH}$ lower value of 5, the incorporation of SPMA is not effective, and the hybrid LDH phase is not formed anymore.

Even if the hybrid material prepared at $\mathrm{pH}$ of 6 may be more suitable than the others for a subsequent delamination, from an academic point of view, we were interested to better understand the process of dispersion of the filler inside the polymer and therefore a crystallized hybrid phase prepared at $\mathrm{pH}$ of 10 was considered in the following.

\subsection{Characterization of nanocomposites SPMA/LDH:PS}

The validity of the polymerization process used was controlled by comparison with a commercially available PS standard. FTIR spectra of the as made PS using 0.1 and $1 \%$ wt of BPO, respectively, and of PSA are displayed in Fig. 2. The three spectra are superimposable, and additional vibration bands are observed for the polymer prepared using $1 \%$ wt of BPO.

The vibrations bands at 1720 and $1270 \mathrm{~cm}^{-1}$ are characteristic of the initiator. These two contributions appear as shoulder for the other as-made polymers.

From the XRD diagrams of the nanocomposites displayed in Fig. 3, several informations can be obtained. Concerning the commercially-available PS, two large humps centered at $2 \theta=10$ and $20^{\circ}$ are characteristic of its semi-crystalline state. These two contributions are observed in the case of the as-made nanocomposites, showing that, 


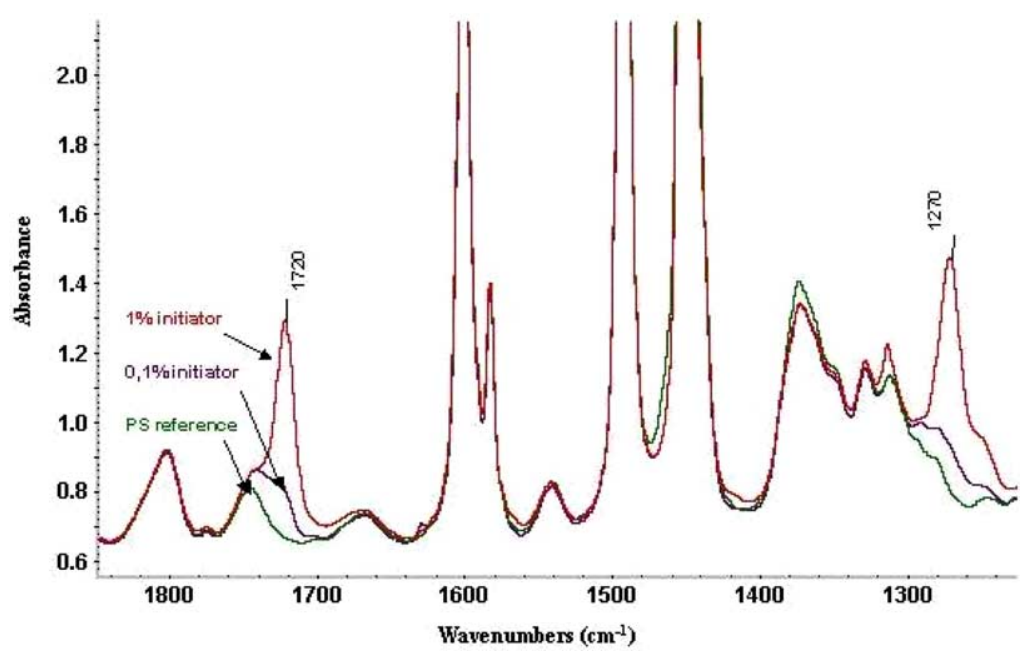

Fig. 2. IR spectra in the region $1900-1200 \mathrm{~cm}^{-1}$ for PS standard, and as made PS prepared with 1 and $0.1 \%$ wt of BPO.

independently of the filler content, a similar crystallinity is reached.

After a thermal treatment, the hybrid filler dispersed into PS is undergoing a dilatation up to $2.52 \mathrm{~nm}$, corresponding to an increase in the basal spacing. Oppositively, when heated, the lamellar structure of the filler SPMA/LDH was found to contract from 1.87 to $1.72 \mathrm{~nm}$ [19].

This may be attributed either to a rearrangement of the organic molecule SPMA or to an incorporation of polymer end-chains inside the gap. The second explanation is more realistic as the state of SPMA present either in the hybrid phase or in the nanocomposite is similar (see below).

In situ polymerization of SPMA within LDH gap occurs at higher temperature $\left(T=160{ }^{\circ} \mathrm{C}\right)$, and was performed in absence of any initiator. The presence of BPO used to initiate the polymerization of styrene may also trigger the polymerization of SPMA. Once again, we should remind that a well-crystallized filler was used, but nevertheless, one can observe the broadening of the harmonics, which indicates that the $2 \mathrm{D}$ character is less and less pronounced.

To give evidence whether SPMA was polymerized simultaneously with the styrene during the achievement of

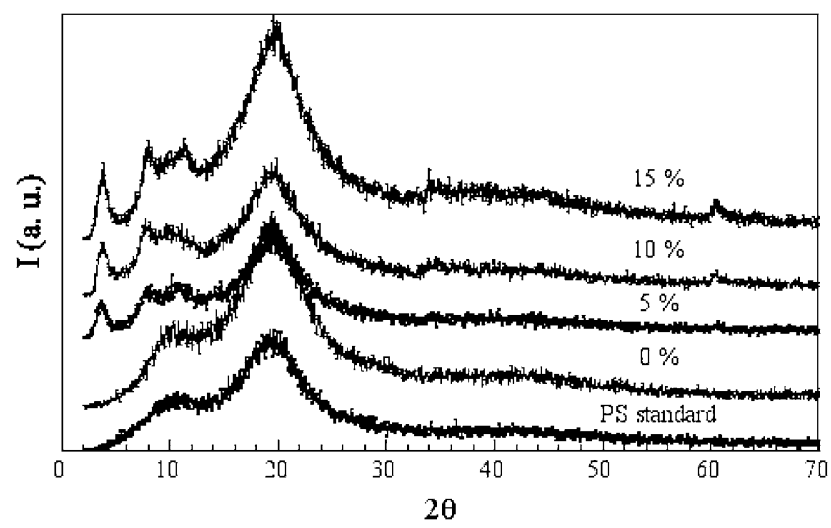

Fig. 3. XRD of PS standard, and of the as-made nanocomposites SPMA/LDH:PS using the ratio (wt $\%$ ) filler to polymer. the nanocomposite, ${ }^{13} \mathrm{C} \mathrm{CP}-\mathrm{MAS}$ high resolution $\mathrm{NMR}$ spectrum of a nanocomposite prepared with a optimum loading of $20 \%$ was compared to that of the filler itself before and after the process of in situ polymerization (Fig. 4). Even if most of the resonance peaks of SPMA are hidden by PS, the change in the resonance peak of carbon nuclei of the methylene group $\left({ }^{3} \mathrm{C}\right)$ may be followed. Before polymerization of SPMA, ${ }^{13} \mathrm{C}$ nuclei response is observed at low field value (18 ppm), whereas after polymerization, the methylene group is then attached to a quaternary carbon, and is down-field shifted. Its contribution has disappeared from the spectrum of SPMA/LDH after polymerization and of the nanocomposite, underlining the fact that SPMA and styrene are concomitantly polymerized.

These results are even more confirmed by the infrared spectra of the various nanocomposites shown in Fig. 5. The characteristic vibration bands of LDH-SPMA are observed,

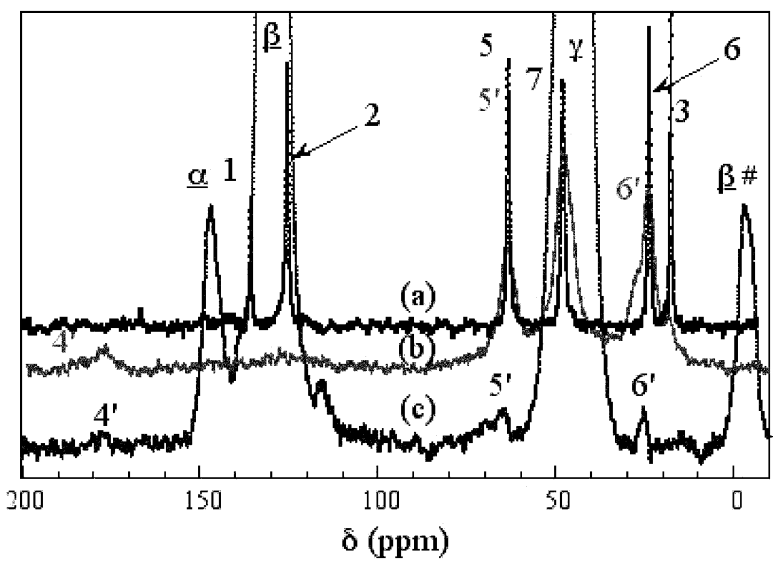

Fig. $4 .{ }^{13} \mathrm{C}$ CP-MAS NMR spectra of the hybrid filler (a) before and (b) after thermal treatment and of (c) the nanocomposite of composition SPMA/LDH:PS equal to 20:80 (\% wt). The labelling is according to SPMA: $\mathrm{H}_{2}^{1} \mathrm{C}={ }^{2} \mathrm{C}\left({ }^{3} \mathrm{CH}_{3}\right)^{4} \mathrm{CO}_{2}^{5} \mathrm{CH}_{2}^{6} \mathrm{CH}_{2}^{7} \mathrm{CH}_{2} \mathrm{SO}_{3}$ and polymerized SPMA: $-\mathrm{H}_{2}^{1^{\prime}} \mathrm{C}^{-2^{\prime}} \mathrm{C}\left({ }^{3^{\prime}} \mathrm{CH}_{3}\right)^{4^{\prime}} \mathrm{CO}_{2}^{5^{\prime}} \mathrm{CH}_{2}^{6^{\prime}} \mathrm{CH}_{2}^{7^{\prime}} \mathrm{CH}_{2} \mathrm{SO}_{3}$, the contribution of PS is designed by grec characters, and the symbol \# denotes spinning side band. 


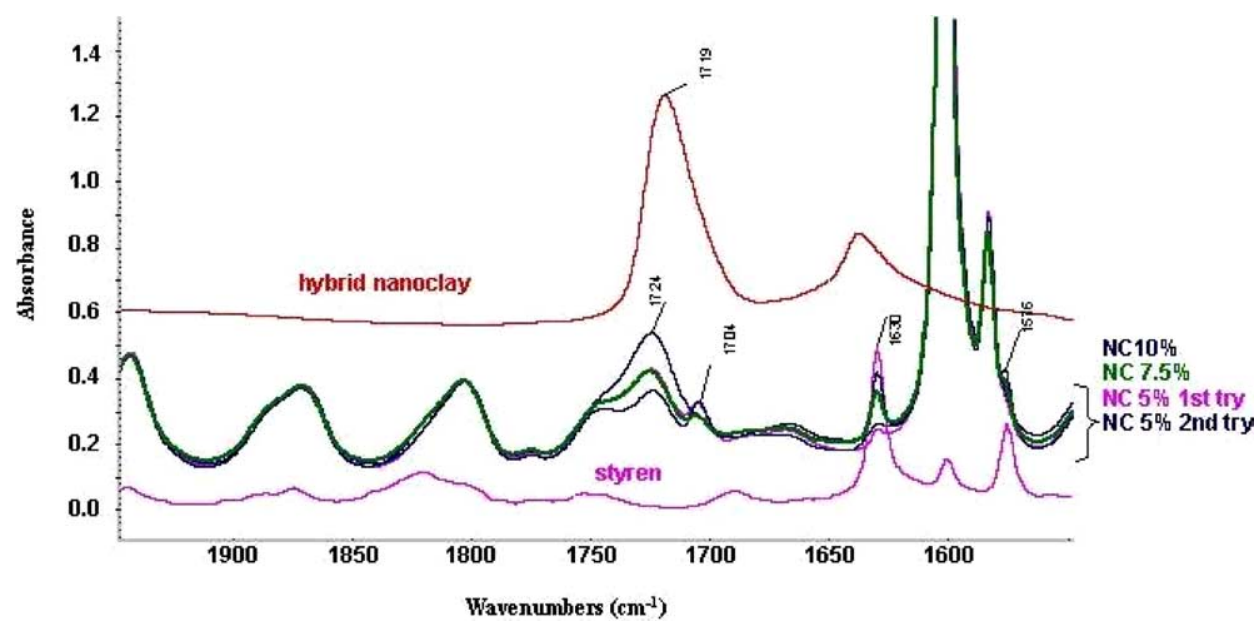

Fig. 5. IR spectra of PS standard, the nanocomposite in the proportion filler to PS of 5, 10, and 15\%, and of the filler alone.

i.e. $\nu(\mathrm{OH}), \nu(\mathrm{C}=\mathrm{O}), \nu(\mathrm{S}=\mathrm{O})$ and $\delta(\mathrm{O}-\mathrm{M}-\mathrm{O})$ at 3478,1720 , 1048 and $425 \mathrm{~cm}^{-1}$, respectively, and in agreement with NMR results, the vibration of the $(\mathrm{C}=\mathrm{C})$ bond which is initially observed at $1638 \mathrm{~cm}^{-1}$ for SPMA is missing on the spectrum of the nanocomposites. The opening of the double band has some effects on other vibration bands such as the $(\mathrm{C}=\mathrm{O})$ one, which is shifted from 1719 to $1724 \mathrm{~cm}^{-1}$. Once again, these results confirm that the in situ polymerization of interleaved SPMA is occurring at much lower temperature in presence of BPO even in small amount.

The infrared spectra also show that it remains non polymerized styrene, as evidenced by the bands at 1630 , 1575,990 , and $775 \mathrm{~cm}^{-1}$. This is confirmed by UV-visible spectroscopy analysis, two absorption maxima at 284 and $292 \mathrm{~nm}$ characteristic of styrene. In the case of the nanocomposites, these bands disappear when the films are submitted to UV-light irradiation. A subsequent thermal treatment at $60{ }^{\circ} \mathrm{C}$ under vacuum for a few days is also able to cure the samples from the presence of styrene.

The dispersion of the LDH-SPMA may be qualitatively estimated from the TEM micrographs (Fig. 6). Qualitat- ively, the stacking of LDH platelets may be numbered between quasi-single layers, visible on the left, to bundles, observed on the right, thus giving the picture for the whole of a mixed intercalated and exfoliated structure. The multiscale dispersion giving rise to object ranging from a few $\mathrm{nm}$ to tenth of $\mathrm{nm}$ may be suitable for nanocomposite reinforcement [1].

Comparatively, monomer surfactant such as styrylammonium molecule was shown to promote exfoliation of montmorillonite into PS or PMMA [20], but in our case the in situ polymerization process was found to be not efficient enough to produce only exfoliated system.

The thermal behavior of the nanocomposites is quite similar to the filler-free polymer up to a loading of $10 \%$ (Fig. 7). This is opposite to recent observations obtained in the case of a nanocomposite filler:PS, with a filler consisting of an organo-modified LDH by dodecyl sulfate [21]. For higher loadings $(>10 \% \mathrm{wt})$, the characteristic features of the hybrid filler (dihydroxylation, departure of the organic molecule) are observed, whereas even for low filler content (5 wt\%), it was observed for DS/LDH:PS [21].
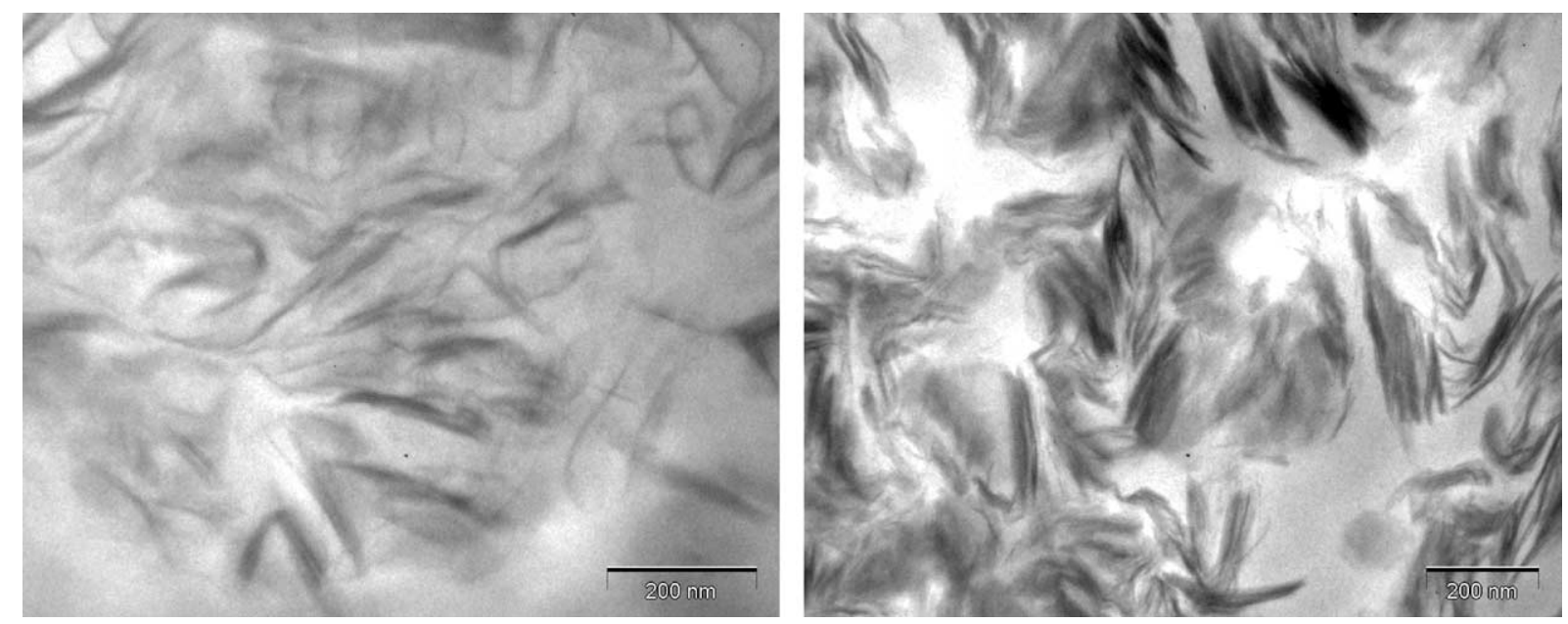

Fig. 6. Transmission electron microscopy micrograph of a nanocomposite based on SPMA/LDH:PS in proportion 10:90 in wt\%. 


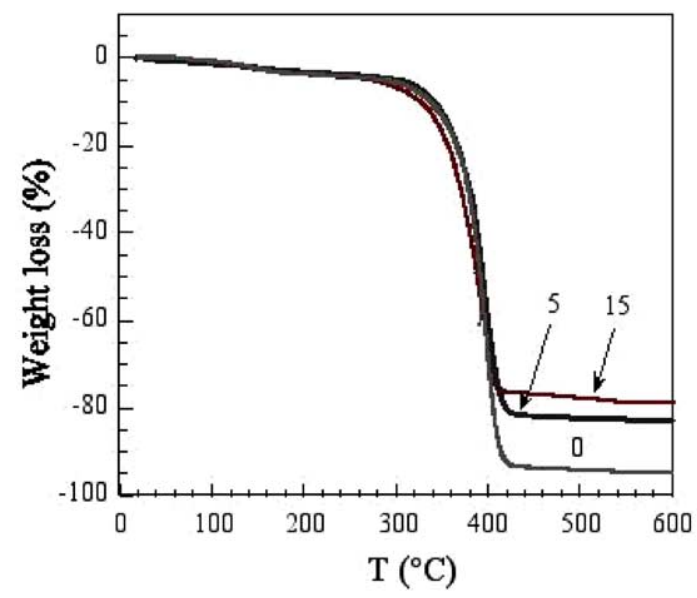

Fig. 7. Thermal analysis of the nanocomposites SPMA/LDH:PS.

Alternatively, the glass transition $T_{\mathrm{g}}$ measured by DSC was found to be quite constant for loadings below $10 \% \mathrm{wt}$, and then was observed to slightly decrease for higher filler loading (Fig. 8). The decrease in $T_{\mathrm{g}}$ can be interpreted by an increase in the mobility of the polymer chains, therefore the filler used in large amount is acting as a plasticizer, as observed for other PS nanocomposites containing montmorillonite [22].

Size exclusion chromatography (SEC) measurements were performed on extracted polymers from the as-made nanocomposites. $M_{\mathrm{w}}$ for commercial PS is of $236,000 \mathrm{~g} \mathrm{~mol}^{-1}$ associated to a polydispersity index of 2.1. For the as-made filler free PS, $M_{\mathrm{w}}$ is found to increase drastically from 40,000 to $370,000 \mathrm{~g} \mathrm{~mol}^{-1}$ ( $I_{\mathrm{p}}$ was almost constant) when the amount of BPO decrease from 1 to $0.1 \%$, respectively. This has to be interpreted with a large number of ignition for the reaction of polymerization, thus decreasing the polymer chain length. For the nanocomposites, $M_{\mathrm{w}}$ is ranging from 340,000 to $430,000 \mathrm{~g} \mathrm{~mol}^{-1}$ with a polydispersity index $I_{\mathrm{p}}$ around 2.1-2.2. This shows clearly that the formation of PS is not impeded by the presence of filler even for high loadings.

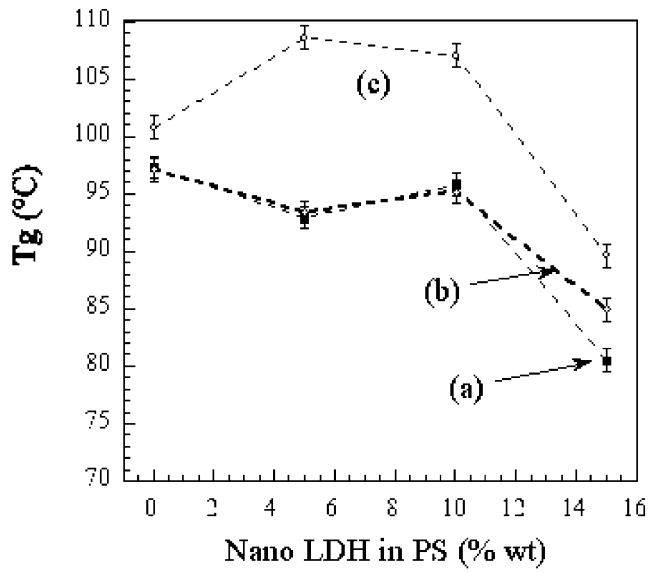

Fig. 8. Tg of the nanocomposite as a function of hybrid LDH content measured during (a) first heating, (b) cooling, and (c) second heating.

\subsection{Study of the photo-oxidation}

UV-light irradiation at $\lambda>300 \mathrm{~nm}$ of polystyrene films leads to changes in the IR spectra in the carbonyl and hydroxyl regions. The IR absorption bands were assigned and the oxidation photoproducts identified which allowed the proposal of a general photooxidation mechanism [17].

In a first time, UV-light exposure of the as-made polystyrene was carried out. In Fig. 9(a), the evolutions of the IR spectra in the region $1900-1500 \mathrm{~cm}^{-1}$ corresponding to the domain of the stretching vibration $\mathrm{C}=\mathrm{O}$ are displayed. Subtraction of the initial spectrum before irradiation from the spectra recorded after several irradiation times allows the observation of the global shape of the broad bands that are formed (Fig. 9(b)). Several maxima or shoulders are observed in the carbonyl region: 1690, 1698, 1704, 1710, 1725 and $1785 \mathrm{~cm}^{-1}$. A broad hydroxyl band was found to form around $3450 \mathrm{~cm}^{-1}$.

Identification of the photoproducts was performed by derivatization reactions with $\mathrm{SF}_{4}$ and $\mathrm{NH}_{3}$ gaz as well as by thermal treatments. The main photoproducts identified were acetophenone and aromatic end-chain ketone $\left(1690 \mathrm{~cm}^{-1}\right)$, benzoic acid $\left(1698 \mathrm{~cm}^{-1}\right)$, benzaldehyde $\left(1704 \mathrm{~cm}^{-1}\right)$, aliphatic acid $\left(1710 \mathrm{~cm}^{-1}\right)$ associated by hydrogen bonding, aliphatic ketone $\left(1725 \mathrm{~cm}^{-1}\right)$ and benzoic anhydride $\left(1725\right.$ and $\left.1785 \mathrm{~cm}^{-1}\right)$. The band at $3450 \mathrm{~cm}^{-1}$ was attributed to hydroperoxides and alcohols associated by hydrogen bonding [16].

These results clearly indicate that the photooxidation mechanism of the as-made polystyrene is identical to the
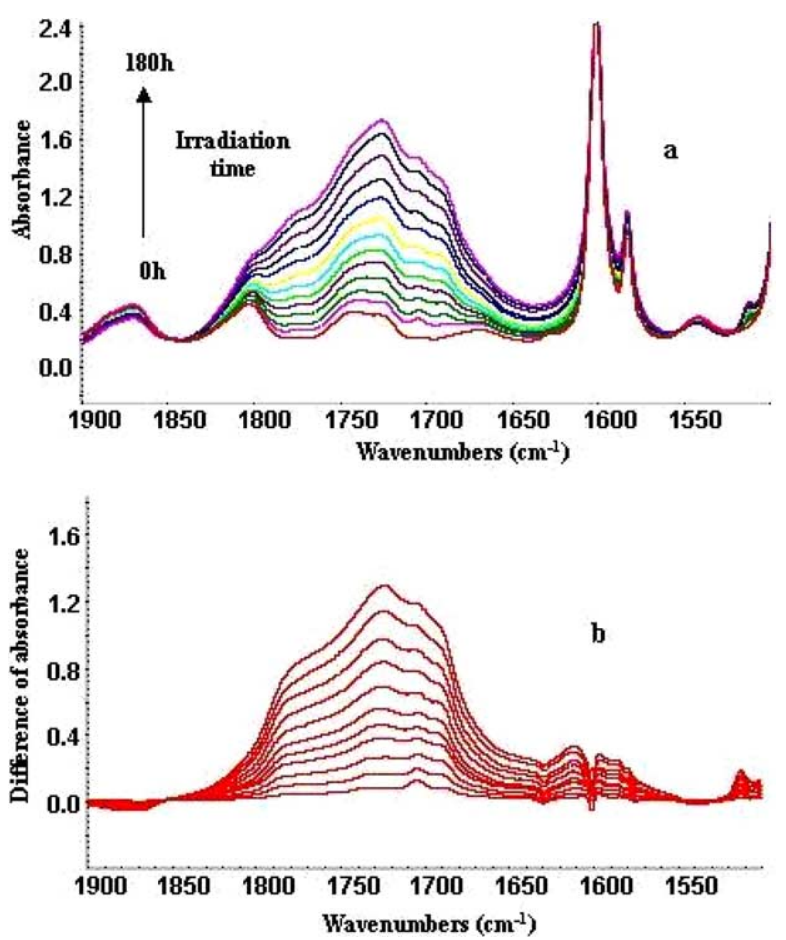

Fig. 9. IR spectra of the as-made PS in the region $1400-1900 \mathrm{~cm}^{-1}$ before (a) and after (b) subtraction of the initial spectrum. 


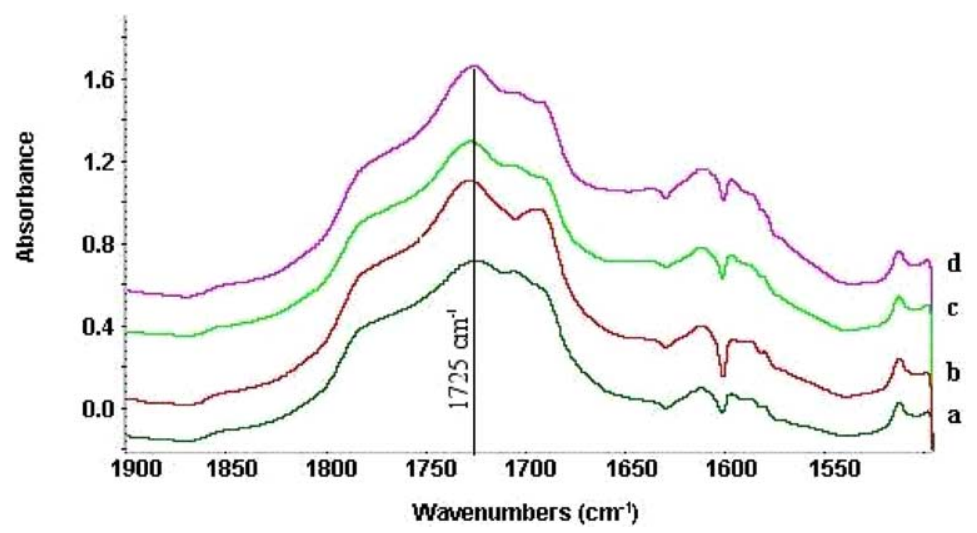

Fig. 10. IR spectra of (a) as-made PS and of the nanocomposites with (b) 5, (c) 10, and (d) $15 \%$ nanoclay loading after an irradiation time of $120 \mathrm{~h}$.

mechanism already reported in the case of 'standard' polystyrene [7]. In a second time, the modifications of the IR spectra of the nanocomposite SPMA/LDH:PS films were recorded. The same modifications during photooxidation as
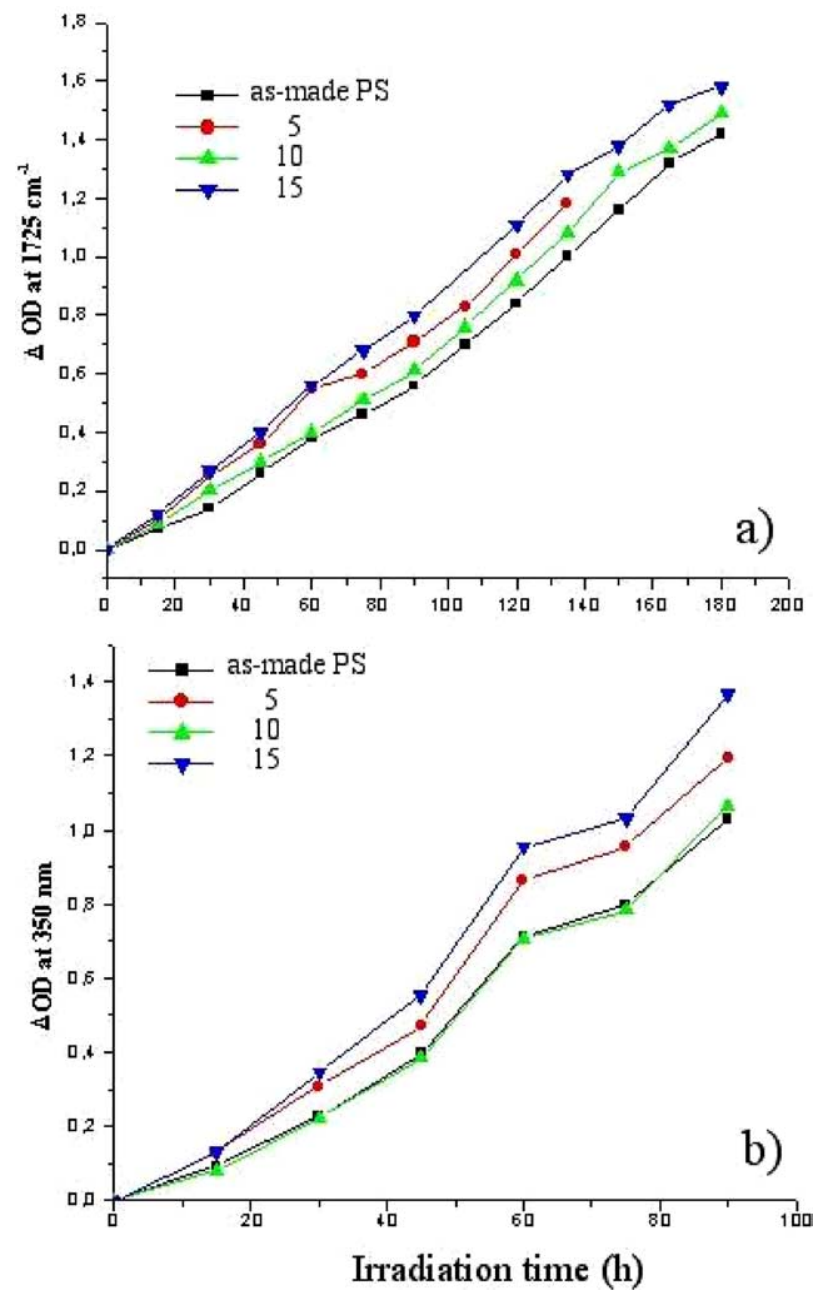

Fig. 11. Variations in (a) IR absorbance at $1725 \mathrm{~cm}^{-1}$, and (b) in optical absorbance at $350 \mathrm{~nm}$ for the as-made PS and the nanocomposite films SPMA/LDH:PS against irradiation time. The percentage of hybrid LDH is indicated. the neat PS (Fig. 10) were observed, which means that similar photoproducts develop in presence of the LDH nanoclay with the same relative concentrations.

\subsection{Rates of photodegradation}

The rates of photooxidation of the nanocomposites containing different amounts of LDH can be compared by measuring the increase in infrared absorbance at $1725 \mathrm{~cm}^{-1}$ and UV absorbance at $350 \mathrm{~nm}$ with irradiation time. Because the various films were slightly different in thickness, the results were multiplied by a correcting factor in order to compare samples with the same amount of PS.

Fig. 11(a) shows the variations of absorbance at $1725 \mathrm{~cm}^{-1}$ versus irradiation time for the pure PS sample and for the nanocomposites SPMA/LDH:PS with different filler contents. One can observe a rate of degradation which is higher in the presence of nanoclay. However, the increase in the oxidation rate does not fit the increase in the amount of $\mathrm{LDH}$, since one can observe that the sample with $5 \%$ degrades slightly faster than the one at $10 \%$.

Fig. 11(b) compares the modifications of the absorbance at $350 \mathrm{~nm}$ during photooxidation. The same conclusions as above can be drawn, with the same peculiar behaviour of PS/LDH nanocomposites with 5\% of the hybrid nanoclay.

\section{Conclusion}

Different compositions of nanocomposite SPMA/LDH:PS were successfully prepared by two-step easily reproducible and environmental quite friendly procedure (no reflux condition in special solvent is required), (i) formation of the nanoclay SPMA/LDH and (ii) dispersion into PS followed by a subsequent polymerization.

As evidenced by XRD, some inorganic layer stackings remain in the nanocomposite, but associated to exfoliated systems vizualized by TEM. Interleaved SPMA was found to polymerize at low temperature in presence of the initiator 
BPO. An increase in the filler content provoks a decrease in $T_{\mathrm{g}}$ for loading higher than $10 \% \mathrm{wt}, \mathrm{SPMA} / \mathrm{LDH}$ is then acting as a plasticizer. At opposite to what was previously thought from reactive filler, the organomodified LDH fillers are, under $10 \% \mathrm{wt}$ in use, not strongly bonded to PS, since they have no effect on the thermal behavior of PS ( $T_{\mathrm{g}}$ and TG).

Finally, the photoxidation study reveals that the hybrid nanofiller does not modify the photooxidation mechanism of PS. The same products of oxidation were observed with the same proportions. A slightly higher oxidation rate was observed in the case of the sample with $5 \%$ of filler.

Similar conclusions were previously obtained with other nanocomposites (PP, epoxy, EPDM and montmorillonite as filler), although an influence of the nanofiller was noted on the efficiency of the stabilizers. The stabilization of SPMA/LDH:PS is the next step of the work. The advantage of this system is his ability to be tailored in order to limit/control eventual interactions with photostabilizers and antioxidants.

\section{Acknowledgements}

We thank Dr Pierre Alcouffe (LMPB-VilleurbanneFrance) for the TEM pictures.

\section{References}

[1] Giannelis EP. Adv Mater 1996;8:29.

[2] Ray SS, Okamoto M. Prog Polym Sci 2003;28:1539.

[3] Pinnavaia TJ, Beall GW. Polymer-clay nanocomposites. New York: Wiley; 2000

[4] Tidjani A, Wilkie CA. Polym Degrad Stab 2001;74:33.

[5] Qin H, Zhao C, Zhang S, Chen G, Yang M. Polym Degrad Stab 2003; 81:497.

[6] Sloan JM, Patterson P, Hsieh A. Polym Mater: Sci Eng 2003;88:354.

[7] Lee HC, Lee TW, Lim YT, Park OO. Appl Clay Sci 2002;21:287.

[8] Morlat S, Mailhot B, Gonzalez D, Gardette JL. Chem Mater 2004;16: 377.

[9] Morlat-Thérias S, Mailhot B, Gonzalez D, Gardette JL. Chem Mater, in press.

[10] Leroux F, Besse JP. Chem Mater 2001;13:3507.

[11] Leroux F, Besse JP. In: Wypych F, Satyanarayana KG, editors. Nanocomposite polymer LDH in clay surfaces: fundamentals and applications. Amsterdam: Elsevier; 2004.

[12] Hsueh HB, Chen CY. Polymer 2003;44:5275.

[13] Hsueh HB, Chen CY. Polymer 2003;44:1151.

[14] Strawhecker KE, Manias E. Chem Mater 2000;12:2943.

[15] Laus M, Camerani M, Lelli M, Sparnacci K, Sandrolini F, Francescangeli O. J Mater Sci 1998;33:2883.

[16] Mailhot B, Gardette JL. Macromolecules 1992;25:4119.

[17] Mailhot B, Gardette JL. Macromolecules 1992;25:4127.

[18] Philippart JL, Sinturel C, Gardette JL. Polym Degrad Stab 1997;58: 261.

[19] Roland-Swanson C, Besse JP, Leroux F. Chem Mater 2004;16:5512.

[20] Wang D, Zhu J, Yao Q, Wilkie CA. Chem Mater 2002;14:3837.

[21] Qiu L, Chen W, Qu B. Polym Degrad Stab 2005;87:433.

[22] Fu X, Qutubuddin S. Mater Lett 2000;42:12. 